\title{
Preconditioning of bone marrow-derived mesenchymal stromal cells by tetramethylpyrazine enhances cell migration and improves functional recovery after focal cerebral ischemia in rats
}

\author{
Lin $\mathrm{Li}^{1}$, Lisheng Chu ${ }^{1 *} \mathbb{D}$, Yan Fang ${ }^{1}$, Yan Yang ${ }^{1}$, Tiebing $\mathrm{Qu}^{1}$, Jianping Zhang ${ }^{2}$, Yuanjun Yin ${ }^{1}$ and Jingjing Gu ${ }^{3}$
}

\begin{abstract}
Background: Transplantation of bone marrow-derived mesenchymal stem cells (BMSCs) is one of the new therapeutic strategies for treating ischemic stroke. However, the relatively poor migratory capacity of BMSCs toward infarcted regions limited the therapeutic potential of this approach. Pharmacological preconditioning can increase the expression of CXC chemokine receptor 4 (CXCR4) in BMSCs and enhance cell migration toward the injury site. In the present study, we investigated whether tetramethylpyrazine (TMP) preconditioning could enhance BMSCs migration to the ischemic brain and improve functional recovery through upregulating CXCR4 expression.

Methods: BMSCs were identified by flow cytometry analysis. BMSCs migration was evaluated in vitro by transwell migration assay, and CXCR4 expression was measured by quantitative reverse transcription-polymerase chain reaction and western blot analysis. In rats with focal cerebral ischemia, the neurological function was evaluated by the modified neurological severity score, the adhesive removal test and the corner test. The homing BMSCs and angiogenesis were detected by immunofluorescence, and expression of stromal cell-derived factor-1 (SDF-1) and CXCR4 was measured by western blot analysis.

Results: Flow cytometry analysis demonstrated that BMSCs expressed CD29 and CD90, but not CD34 and CD45. TMP pretreatment dose-dependently induced BMSCs migration and CXCR4 expression in vitro, which was significantly inhibited by AMD3100, a CXCR4 antagonist. In rat stroke models, we found more TMP-preconditioned BMSCs homing toward the infarcted regions than nonpreconditioned cells, leading to improved neurological performance and enhanced angiogenesis. Moreover, TMP-preconditioned BMSCs significantly upregulated the protein expression of SDF-1 and CXCR4 in the ischemic boundary regions. These beneficial effects of TMP preconditioning were blocked by AMD3100.

Conclusion: TMP preconditioning enhances the migration and homing ability of BMSCs, increases CXCR4 expression, promotes angiogenesis, and improves neurological performance. Therefore, TMP preconditioning may be an effective strategy to improve the therapeutic potency of BMSCs for ischemic stroke due to enhanced BMSCs migration to ischemic regions.
\end{abstract}

Keywords: Bone marrow-derived mesenchymal stem cells, Tetramethylpyrazine, Preconditioning, Migration, CXCR4

\footnotetext{
* Correspondence: chulisheng@21cn.com

'Department of Physiology, Zhejiang Chinese Medical University, Hangzhou

310053, China

Full list of author information is available at the end of the article
} 


\section{Background}

Stroke is a major cause of death and adult disability worldwide. Currently, administration of recombinant tissue plasminogen activator is the sole effective treatment for acute ischemic stroke. However, only approximately $5 \%$ of the patients could benefit from this treatment due to the narrow therapeutic time window and the risk of subsequent hemorrhage [1]. Thus, developing restorative therapeutics, particularly stem cell therapy, has attracted great interest [2, 3]. Bone marrow-derived mesenchymal stem cells (BMSCs) represent an ideal candidate for cell transplantation therapy because they are easily obtained and can be expanded rapidly ex vivo [4]. BMSCs transplantation is reported to be safe and effective for the treatment of ischemic stroke [5-7]. However, the low migration efficiency of the transplanted BMSCs toward the injured area limits the effect of BMSC delivery via the vascular route $[8,9]$. Thus, strategies promoting BMSCs migration into the peri-ischemic brain tissue may enhance the effectiveness of BMSCs-based therapy [10].

Chemokines play a pivotal role in controlling cellular migration. It has been proven that stromal cell-derived factor-1 (SDF-1) contributes largely to recruitment of stem cells to ischemic tissue by combining with its receptor CXC chemokine receptor 4 (CXCR4) [11, 12]. SDF1 is upregulated in the ischemic penumbral region after stroke, and the interaction between SDF-1 and CXCR4 triggers BMSCs migration toward the injured area [13-16]. While CXCR4 is highly expressed in BMSCs within the bone marrow, its expression is severely reduced during ex vivo expansion of BMSCs [17, 18]. Such low levels of CXCR4 could decrease the ability of transplanted BMSCs to respond to homing signals emanated from the ischemic tissue. Most MSCs have high intracellular CXCR4 expression, yet only a small percentage of MSCs express CXCR4 on the cell surface [19]. Therefore, how to mobilize the internalized receptor and increase membranous CXCR4 expression becomes critical to improving the engraftment of BMSCs and the benefit of their transplantation. Some studies have shown that cell migration could be improved by preconditioning the stem cells with chemical compounds, cytokines, and hypoxic conditions during the stage of in vitro expansion before transplantation [10].

Tetramethylpyrazine (TMP), a pharmacologically active component extracted from the rhizome of the Chinese herb Rhizoma Chuanxiong (Chuanxiong), has been widely used in clinical treatment of cerebrovascular and cardiovascular diseases in China [20-22]. However, the molecular mechanisms involved in the therapeutic effects of TMP are largely unclear. Recently, TMP was found to act as a highly efficient regulator for the migration of various cell types, such as neural precursor cells [23], bone marrow mononuclear cells [24], and brain endothelial cells [25]. These finding suggest that TMP may have the potential to promote the migration of BMSCs. In the present study, we studied the effect of TMP preconditioning on BMSCs migration and homing followed by exploration of the relevant mechanisms, and evaluated the effect of TMP-preconditioned BMSCs on the neurological function and angiogenesis after ischemic stroke in rats.

\section{Methods}

\section{Animals}

Male Sprague-Dawley rats were purchased from SinoBritish SIPPR/BK Laboratory Animal (Shanghai, China), and were housed at the Laboratory Animal Research Center of Zhejiang Chinese Medical University on a 12-h light/dark cycle with free access to water and food. All animal procedures were approved by the Experimental Animal Ethics Committee of Zhejiang Chinese Medical University (reference number: ZSLL-2014-37), Hangzhou, China and were conducted in accordance with the Guidelines for the Care and Use of Laboratory Animals established by the Science and Technology Committee of Zhejiang Province.

\section{Isolation, culture, and identification of BMSCs}

BMSCs were harvested from 3-week-old Sprague-Dawley rats weighing 80-100 g. Briefly, the bone marrow was flushed from the femurs and tibias of rats with DMEM/F12 (Gino Bio-Pharm Technology, Hangzhou, China), 1\% (v/v) penicillin and streptomycin (Haotian Biological Technology, Hangzhou, China). The cells were centrifuged and suspended in DMEM/F12 with 10\% fetal bovine serum (FBS) (Gibco, Thermo Fisher Scientific, MD, USA), and cultured in $5 \% \mathrm{CO}_{2}$ at $37{ }^{\circ} \mathrm{C}$. After $48 \mathrm{~h}$ of incubation, the medium was changed and replaced every 3 days thereafter. When BMSCs reached 80-90\% confluence, they were digested with $0.05 \%$ trypsin-EDTA (Gino Bio-Pharm Technology) and were subcultured at a ratio of 1:2. BMSCs from passage 3 were used in all experiments.

For identification of BMSCs, cell surface markers were analyzed by flow cytometry (Beckman-Coulter Inc., CA, USA). The cells were incubated with fluorescein isothiocyanate-conjugated antibodies against CD90, CD29, CD45 (Biolegend, San Diego, CA, USA), CD34 (Santa Cruz, CA, USA) and PBS (negative control) in a black chamber at $4{ }^{\circ} \mathrm{C}$ for $30 \mathrm{~min}$. At least $5 \times 10^{5}$ cells per sample were acquired and analyzed.

\section{Preconditioning of BMSCs with TMP in vitro}

When BMSCs reached 70-80\% confluence, the cells were pretreated with gradient concentrations of TMP (Aladdin, Shanghai, China) $(0,10,25,50,100$, and $200 \mu \mathrm{M})$ for $24 \mathrm{~h}$ under a $37^{\circ} \mathrm{C}$ and $5 \% \mathrm{CO}_{2}$ environment.

\section{Transwell migration assay}

The migration assay was performed using transwell plates with pore size of $8 \mu \mathrm{m}$ (Corning Costar, Cambridge, 
MA, USA). The upper chambers were loaded with $8 \times$ $10^{4}$ BMSCs in $200 \mu \mathrm{l}$ of DMEM/F12 containing $5 \%$ FBS. The lower chambers were loaded with $600 \mu \mathrm{l}$ of DMEM/F12 containing 5\% FBS, and $100 \mathrm{ng} / \mathrm{ml} \mathrm{SDF-1}$ (Peprotech Inc., Rocky Hill, NJ, USA). The chambers were incubated at $37{ }^{\circ} \mathrm{C}$ and $5 \% \mathrm{CO}_{2}$ for $8 \mathrm{~h}$. The upper surface of the membrane was then gently scraped to remove the nonmigrated cells and washed with PBS. The membrane was then fixed in $4 \%$ paraformaldehyde for $10 \mathrm{~min}$ followed by staining with $0.5 \%$ crystal violet for $20 \mathrm{~min}$. The number of migrated cells was determined by averaging five random fields per well. The experiments were performed in quadruplicate for each group. For inhibitor studies, BMSCs were treated with or without TMP, and then were incubated for $8 \mathrm{~h}$ with $100 \mu \mathrm{g} /$ ml AMD3100 (Sigma Aldrich, St Louis, MO, USA), a specific antagonist of CXCR4.

\section{Quantitative reverse transcription-polymerase chain reaction}

Total RNA was extracted from brain tissue using TRIzol Reagent (Invitrogen, Carlsbad, CA, USA) according to the manufacturer's instructions. cDNA was produced from the total RNA using PrimeScript RT Master Mix (TaKaRa, Tokyo, Japan). Quantitative PCR was conducted using the SYBR Premix Ex Taq Kit (TaKaRa) on an iQ5 multiplex real-time fluorescence quantitative PCR instrument (Bio-Rad, Hercules, CA, USA). Primers for CXCR4 consisted of $5^{\prime}$-ACT CAA TTC CAT GAG CAG AG-3' (forward) and 5'-CTT TGC GTA AGT GTT AGC TG-3' (reverse). Primers for GAPDH (an internal control) consisted of 5' -ACA GCA ACA GGG T GG TGG AC-3' (forward) and 5'-TTT GAG GGT GCA GCG AAC TT-3' (reverse). The reaction conditions were as following: preheated at $95{ }^{\circ} \mathrm{C}$ for $5 \mathrm{~min}$, followed by 40 cycles of denaturation at $95{ }^{\circ} \mathrm{C}$ for $30 \mathrm{~s}$, annealing at $55{ }^{\circ} \mathrm{C}$ for $30 \mathrm{~s}$, and extension at $72{ }^{\circ} \mathrm{C}$ for $30 \mathrm{~s}$. Each sample was tested in triplicate, and relative gene expression data were analyzed using the $2^{-\Delta \Delta C T}$ method.

\section{Western blot analysis}

The total protein concentration of cells or tissue was analyzed using the bicinchoninic acid assay (BCA; Beyotime, Beijing, China) according to the manufacturer's instructions. Proteins were electrophoresed on a sodium dodecyl sulfate (SDS) $-10 \%$ polyacrylamide gel and were transferred onto polyvinylidene difluoride (PVDF) membranes (Bio-Rad). Subsequently, membranes were incubated at $4{ }^{\circ} \mathrm{C}$ overnight with primary antibodies of rabbit anti-CXCR4 (Santa Cruz), anti-SDF-1 (Santa Cruz), or mouse anti- $\beta$-actin antibody (Santa Cruz), followed by incubation with goat anti-rabbit IgG (Thermo Pierce, USA) or goat anti-mouse IgG (Thermo Pierce) secondary antibody at room temperature for $2 \mathrm{~h}$. The membrane was visualized using a chemiluminescence enhanced detection kit (Thermo Pierce), and was exposed to X-ray films. The expression of CXCR4 and SDF-1 was then normalized to $\beta$-actin.

\section{Middle cerebral artery occlusion in rats}

The middle cerebral artery occlusion (MCAO) was performed using a modified method initially described by Longa et al. [26]. Briefly, rats were anesthetized with $10 \%$ chloral hydrate $(350 \mathrm{mg} / \mathrm{kg}$ body weight) via intraperitoneal injection. The right carotid artery, external carotid artery (ECA), and internal carotid artery (ICA) were carefully isolated. The ECA was ligated and cut with a small mouth. A 4-0 nylon was inserted through the right ECA and was gently advanced into the ICA up to a point approximately $18 \pm 2 \mathrm{~mm}$ from the bifurcation. After occlusion for $90 \mathrm{~min}$, the nylon suture was withdrawn to permit reperfusion. Sham-operated rats underwent the same procedure without the nylon suture. A heating lamp was used to maintain the rectal temperature around $37^{\circ} \mathrm{C}$ during the surgical procedure.

\section{BMSCs labeling and cell transplantation}

Before transplantation, BMSCs were incubated with $10 \mu \mathrm{mol} / \mathrm{L} \mathrm{BrdU}$ (Sigma Aldrich) for $48 \mathrm{~h}$. BMSCs were then preconditioned with $100 \mu \mathrm{M}$ TMP for $24 \mathrm{~h}$ and with $20 \mu \mathrm{M}$ AMD3100 for $6 \mathrm{~h}$ [27]. Twenty-four hours after MCAO, approximately $1 \times 10^{6}$ BMSCs in $1 \mathrm{ml}$ PBS were injected into the rat via the tail vein. The rats were then randomly divided into five groups $(n=12$ rats per group): sham-operated control group; MCAO rats with injection of PBS (MCAO group); MCAO rats with transplantation of nonpreconditioned BMSCs (BMSC group); MCAO rats with transplantation of TMPpreconditioned BMSCs (TMP group); and MCAO rats with transplantation of TMP/AMD3100-preconditioned BMSCs (AMD3100 group).

\section{Neurological function evaluation}

The neurological deficits were evaluated 1,7 , and 14 days after ischemia by the investigators who were blinded to the experimental design. The modified neurological severity score (mNSS) includes motor, sensory, reflex, and balance tests [7]. The mNSS was used to evaluate the sensorimotor deficits by grading the score on a scale of 0-18 (normal score, 0; maximal deficit score, 18). The inability to perform a test or the lack of a tested reflex scores one point. Thus, a higher score means higher severity of injury. The adhesive removal test was used to assess somatosensory deficit as described previously [7]. Briefly, two small pieces of adhesive-backed paper dots (of equal size, $113.1 \mathrm{~mm}^{2}$ ) were used as bilateral tactile stimuli occupying the distal-radial area on the wrist of 

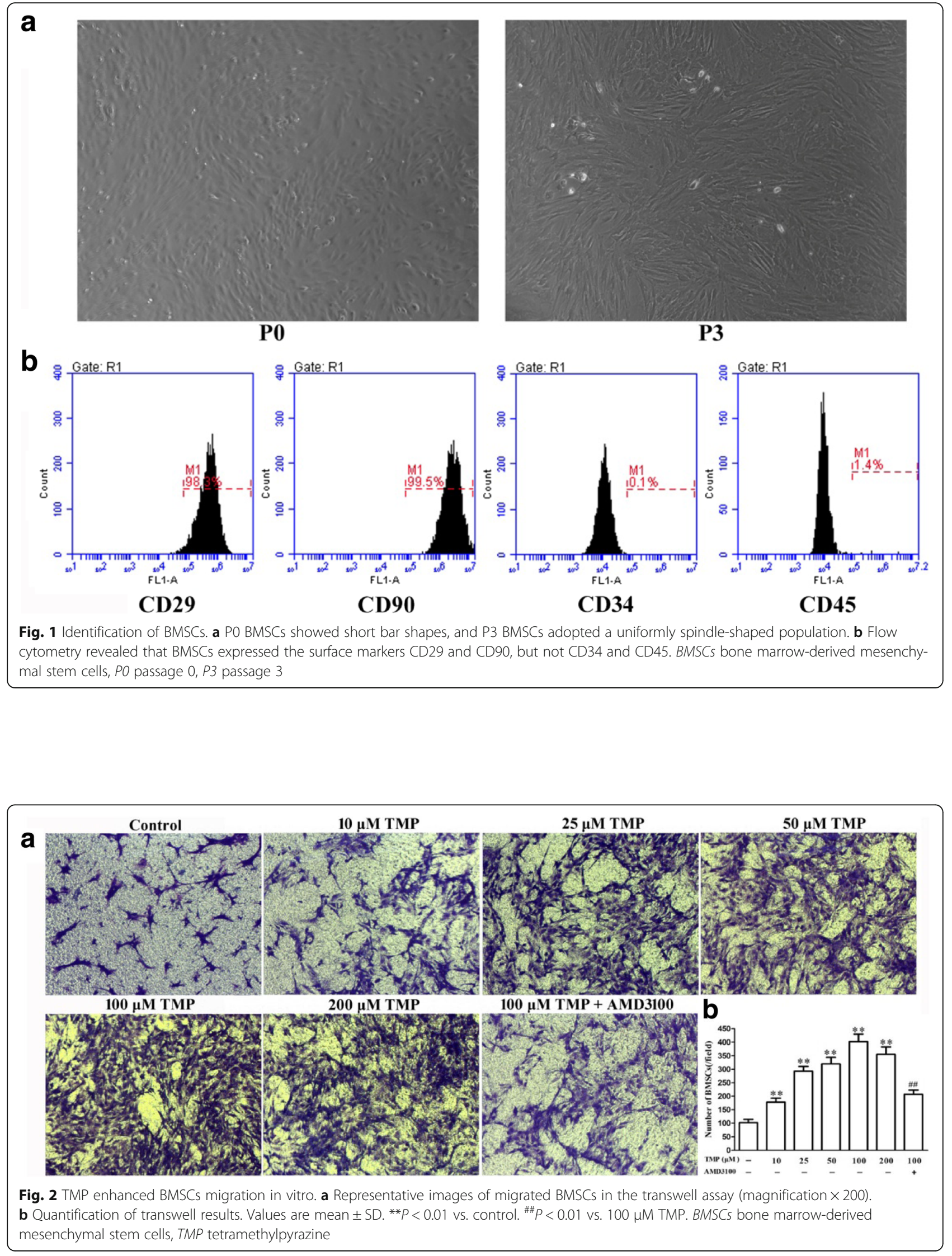
each forelimb. The time to remove each stimulus from the forelimbs was recorded in five trials per day for each forepaw. After 3 consecutive days of training, all of the rats were able to remove the dots within $10 \mathrm{~s}$ and then were subjected to MCAO. The mean time to remove the left dot was recorded. Sensorimotor function was also evaluated by the corner test which involves both stimulations of the vibrissae (sensory/neglect) and rearing (motor response) [28]. Briefly, two boards $(30 \mathrm{~cm} \times 20 \mathrm{~cm} \times 1 \mathrm{~cm})$ were set at a $30^{\circ}$ angle with a small opening along the joint. A rat was placed between two boards facing the corner. When the rat entered deeply toward the corner, the two boards together stimulated both sides of the vibrissae. The rat then reared forward and upward, after which it turned back to face the open end. Ten trials were performed for each rat, and the selected sides for turning were noted.

\section{Immunofluorescence staining}

The rats were sacrificed 14 days after MCAO by an overdose of $10 \%$ chloral hydrate. The rats were perfused transcardially with ice-cold saline, and then fixed by perfusion with $4 \%$ paraformaldehyde solution. The brains were removed and kept in 4\% paraformaldehyde solution overnight at $4{ }^{\circ} \mathrm{C}$, and then soaked in $30 \%$ sucrose solution. The brain tissue was cut into $10-\mu$ m-thick frozen sections (Leica CM1950; Leica Microsystems, Wetzlar, Germany). BrdU-labeled BMSCs were detected by immunofluorescence staining. Briefly, the sections were first pretreated to denature DNA as follows: after immersion in $50 \%$ formamide/ $2 \times$ saline-sodium citrate buffer (SSC) at $65{ }^{\circ} \mathrm{C}$ for $2 \mathrm{~h}$, the sections were washed in PBS for $10 \mathrm{~min}$, incubated in $2 \mathrm{~N} \mathrm{HCl}$ at $37{ }^{\circ} \mathrm{C}$ for $30 \mathrm{~min}$, and washed with $0.1 \mathrm{M}$ boric acid $(\mathrm{pH} 8.5)$ for $10 \mathrm{~min}$. The sections were then incubated with a mouse anti-BrdU (1:100; Sigma Aldrich) at $4{ }^{\circ} \mathrm{C}$ overnight, washed in PBS, and then incubated with secondary antibodies of Alexa Fluor 555-conjugated goat antimouse IgG (Thermo Fisher Scientific Inc., MA, USA) for $1 \mathrm{~h}$ at $37{ }^{\circ} \mathrm{C}$. Counterstaining was done with 4',6diamidino-2-phenylindole (DAPI; Zhongshan Golden Bridge Biotechnology, Beijing, China). For angiogenesis analysis, the tissue sections were washed twice in PBS and incubated in $0.3 \%$ Triton $\mathrm{X}-100$ for $30 \mathrm{~min}$, incubated in $3 \% \mathrm{H}_{2} \mathrm{O}_{2}$ for $20 \mathrm{~min}$ and then in $5 \%$ goat serum for $1 \mathrm{~h}$, and stained with rabbit anti-von Willebrand factor (vWF; Santa Cruz). Fluorescein isothiocyanate (FITC)-conjugated goat anti-rabbit IgG (Santa Cruz) was used as the secondary antibody.

\section{Statistical analysis}

Data were presented as mean \pm standard deviation (SD) and were analyzed using SPSS software (version 17.0; SPSS Inc., Chicago, IL, USA). mNSS was analyzed by nonparametric Mann-Whitney $U$ test. One-way analysis of variance followed by the Tukey's post-hoc test was used for analyzing parametric data and multiple comparison, and $P$ $<0.05$ was considered statistically significant.

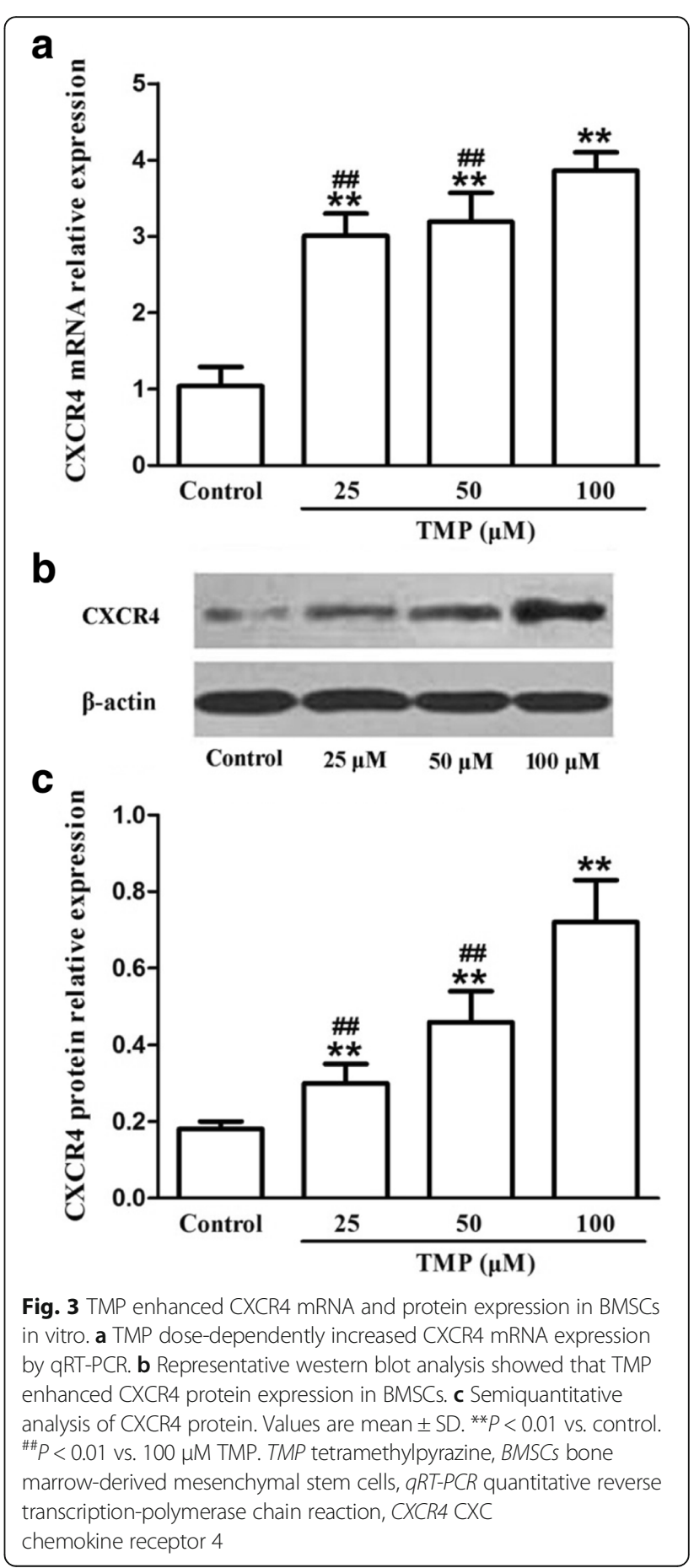




\section{Results}

\section{Identification of BMSCs}

The primary BMSCs cultured in the culture dish displayed short bar shapes initially. Passage 3 (P3) BMSCs showed typical spindle-shaped cell morphology (Fig. 1a). Flow cytometry analysis confirmed that these cells were positive for mesenchymal cell markers CD29 (98.3\%) and CD90 (99.5\%), but negative for hematopoietic cell markers CD34 (0.1\%) and CD45 (1.4\%) (Fig. 1b), suggesting that the cultured cells had similar morphological and immunophenotypical characteristics of BMSCs.

\section{Preconditioning with TMP enhances BMSCs migration in vitro}

A transwell system was used to investigate the effect of TMP preconditioning on BMSCs migration in vitro. Using SDF-1 as a chemotactic agent in the lower chamber, preconditioning with $\operatorname{TMP}(0,10,25,50,100$, and $200 \mu \mathrm{M})$ for $24 \mathrm{~h}$ concentration-dependently increased the BMSCs migratory capacity, especially in the $100 \mu \mathrm{M}$ TMP group (Fig. 2). Most importantly, cotreatment of BMSCs with AMD3100 $(100 \mu \mathrm{g} / \mathrm{ml})$, a CXCR4 antagonist, significantly inhibited the effects of TMP on cell mobility (Fig. 2). These results indicated that preconditioning with TMP promoted BMSCs migration through SDF-1/CXCR4 pathway.

\section{Preconditioning with TMP enhances CXCR4 expression in BMSCs}

To test whether TMP preconditioning could enhance BMSCs migration via upregulating CXCR4 expression, the CXCR4 expression was detected by quantitative reverse transcription-polymerase chain reaction (qRT-PCR) and western blot analysis. The results showed that TMP preconditioning for $24 \mathrm{~h}$ significantly increased CXCR4
mRNA (Fig. 3a) and protein (Fig. 3b, c) expression in BMSCs in a concentration-dependent manner. These data demonstrated that TMP enhanced BMSCs migration by upregulating the expression of CXCR4.

\section{Transplantation of BMSCs preconditioned with TMP improves neurological function recovery}

To determine whether BMSCs preconditioned with TMP $(100 \mu \mathrm{M})$ improved neurological function recovery, the mNSS, the adhesive removal test, and the corner test were performed 1,7 , and 14 days after MCAO. BMSCs preconditioned with TMP significantly improved the functional outcome compared with the BMSC group at 7 and 14 days (Fig. 4), respectively. However, these beneficial effects of TMP preconditioning were reduced by AMD3100 (Fig. 4a-c).

\section{Preconditioning with TMP enhances BMSCs homing into the ischemic brain}

To detect the homing efficiency of injected BMSCs, the number of BrdU-positive BMSCs was counted in the ischemic regions. In the penumbra cortex, TMP preconditioning increased BMSCs homing efficiency compared with the BMSCs group on day 14 after MCAO (Fig. 5a, b). However, copreconditioning with the CXCR4 antagonist AMD3100 largely inhibited the effects of TMP (Fig. 5a, b).

\section{Transplantation of BMSCs preconditioned with TMP increases angiogenesis in ischemic regions}

Microvessel density was analyzed in the penumbra cortex on day 14 after MCAO. Compared with the MCAO group, BMSCs transplantation significantly increased microvessel density. Moreover, MCAO rats transplanted
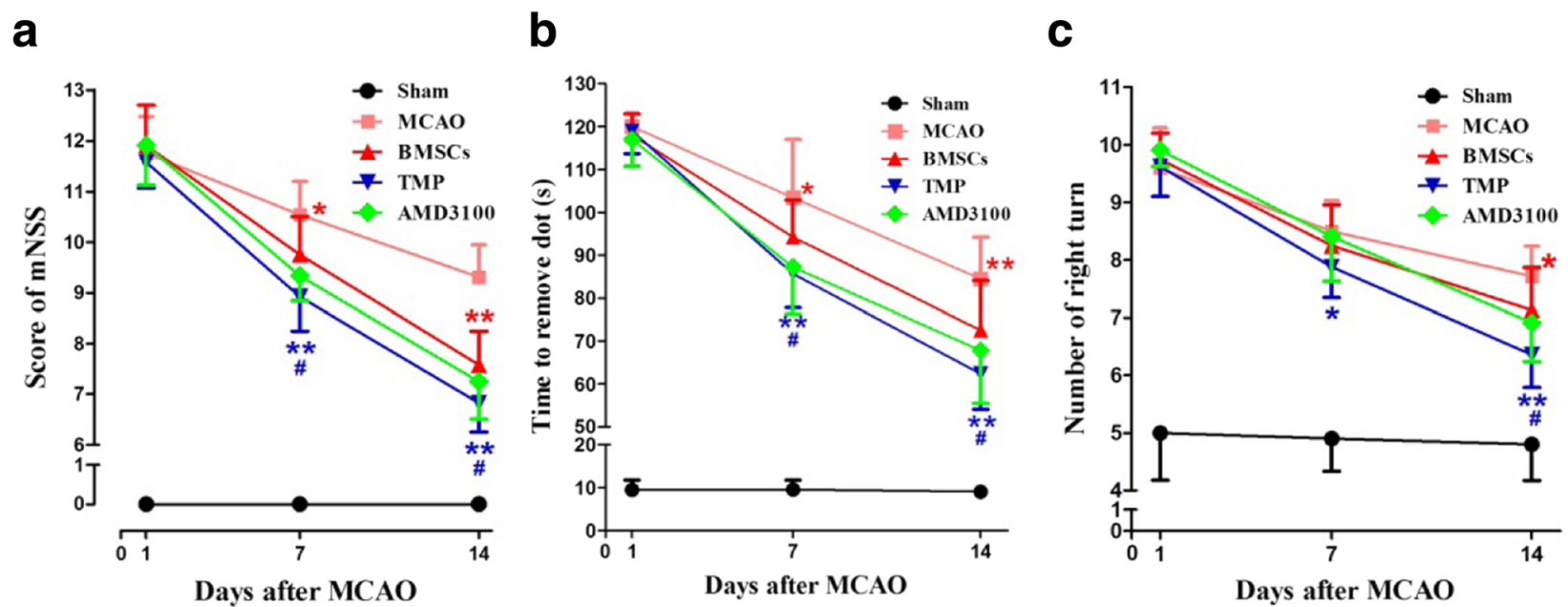

Fig. 4 BMSCs preconditioned with TMP improved functional recovery in MCAO rats. a mNSS test. b Adhesive removal test. c Corner test. ${ }^{*} P<0.05,{ }^{* *} P<0.01$ vs. MCAO. \#P $<0.05$ vs. AMD3100. BMSCs bone marrow-derived mesenchymal stem cells, MCAO middle cerebral artery occlusion, mNSS modified neurological severity scores, TMP tetramethylpyrazine 


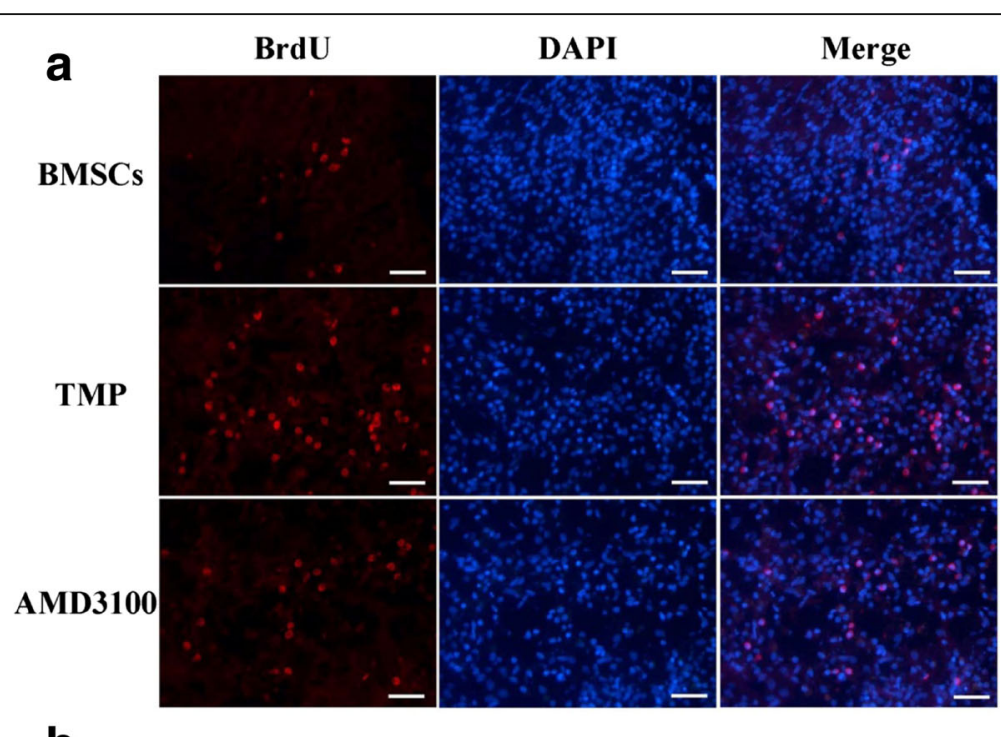

b

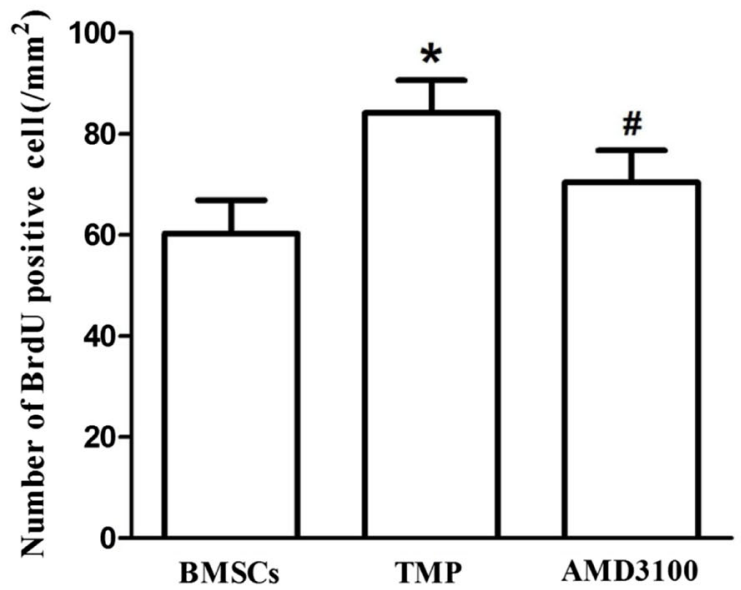

Fig. 5 BMSCs preconditioned with TMP enhances BMSCs homing into the ischemic brain region. a BMSCs that were labeled by BrdU (red) and nuclei labeled with DAPI (blue) were observed at the lesion site after MCAO. b Quantification of migrated BMSCs. Bars, $100 \mu \mathrm{m}$. Values are mean \pm SD. ${ }^{*} P<0.01$ vs. BMSCs. $\# P<0.01$ vs. TMP. BMSCs bone marrow-derived mesenchymal stem cells, TMP tetramethylpyrazine, MCAO middle cerebral artery occlusion, BrdU 5-bromo-2-deoxyuridine, DAPI 4,6-diamidino-2-phenylindole (Color figure online)

with BMSCs preconditioned with TMP had the highest microvessel density (Fig. 6a, b). However, copreconditioning with AMD3100 partially eliminated the effects of TMP (Fig. 6a, b). These data indicated that TMPpreconditioned BMSCs increased angiogenesis in the ischemic regions.

Transplantation of BMSCs preconditioned with TMP promotes protein expression of SDF-1 and CXCR4 in ischemic regions

The protein levels of SDF-1 and CXCR4 were detected by western blot analysis. Expression of SDF-1 and CXCR4 was significantly increased in the BMSC and TMP-preconditioned groups compared with the MCAO group (Fig. 7a, b). Compared with the BMSC group, the protein expression levels of SDF-1 and CXCR4 were significantly increased in the TMP-preconditioned group (Fig. 7a, b). These data indicated that TMP preconditioning stimulated the SDF-1/CXCR4 axis and enhanced BMSCs migration to the ischemic regions.

\section{Discussion}

In the present study, we found that TMP enhanced BMSCs migration and increased CXCR4 mRNA and protein expression in vitro. We then demonstrated that TMP preconditioning before transplantation improved BMSCs homing toward ischemic brain after MCAO treatment, which was accompanied by improved behavioral performance and angiogenesis in the penumbra cortex. However, these effects were largely abolished by the CXCR4 antagonist AMD3100, indicating that the benefits of TMP preconditioning are mediated by the SDF-1/CXCR4 axis. To the best of our knowledge, the current study is the first 

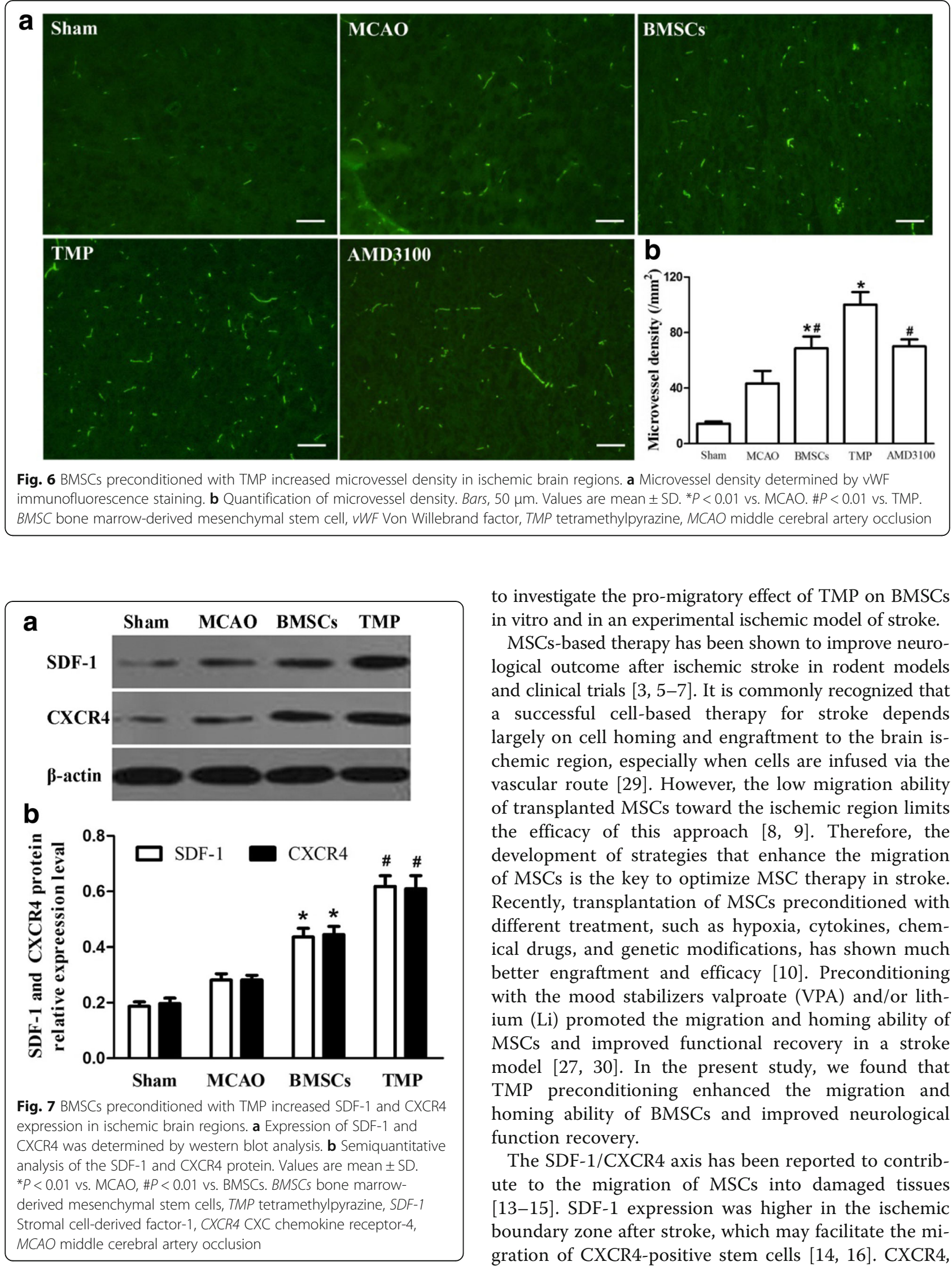

to investigate the pro-migratory effect of TMP on BMSCs in vitro and in an experimental ischemic model of stroke.

MSCs-based therapy has been shown to improve neurological outcome after ischemic stroke in rodent models and clinical trials $[3,5-7]$. It is commonly recognized that a successful cell-based therapy for stroke depends largely on cell homing and engraftment to the brain ischemic region, especially when cells are infused via the vascular route [29]. However, the low migration ability of transplanted MSCs toward the ischemic region limits the efficacy of this approach [8,9]. Therefore, the development of strategies that enhance the migration of MSCs is the key to optimize MSC therapy in stroke. Recently, transplantation of MSCs preconditioned with different treatment, such as hypoxia, cytokines, chemical drugs, and genetic modifications, has shown much better engraftment and efficacy [10]. Preconditioning with the mood stabilizers valproate (VPA) and/or lithium (Li) promoted the migration and homing ability of MSCs and improved functional recovery in a stroke model $[27,30]$. In the present study, we found that TMP preconditioning enhanced the migration and homing ability of BMSCs and improved neurological function recovery.

The SDF-1/CXCR4 axis has been reported to contribute to the migration of MSCs into damaged tissues [13-15]. SDF-1 expression was higher in the ischemic boundary zone after stroke, which may facilitate the migration of CXCR4-positive stem cells $[14,16]$. CXCR4, 
the specific receptor of SDF-1, is highly expressed in BMSCs in the bone marrow. However, its expression is markedly reduced in culture-expanded BMSCs, leading to decreased migration toward the SDF-1 gradient $[19,31]$. Thus, approaches to increase the expression of CXCR4 can enhance the migration ability of BMSCs. Genetic approach is an effective method to increase the expression of CXCR4 in MSCs. CXCR4 overexpression with lentiviral transduction in the MSCs promoted their migration and enhanced neuroprotection in a rat model of cerebral ischemia [32]. However, genetic modification seems infeasible in current clinical practice. Unlike genetic engineering approaches, pharmacological preconditioning is a simple and clinically feasible approach. Tsai et al. $[27,30]$ demonstrated that preconditioning with the mood stabilizer valproic acid (VPA) promoted the homing and migration ability of MSCs through upregulation of CXCR4 expression. Similarly, treatment with tanshinone IIA and astragaloside IV enhanced the ability of MSCs to home to ischemic myocardium sites by increasing the expression of CXCR4 [33]. TMP is one of the most important active components of a traditional Chinese herb from Ligustium wallichii Franchat, which has been widely used in China for the treatment of ischemic stroke [20-22]. Consistent with previous studies [23-25], we found that increased CXCR4 expression with TMP pretreatment enhanced the migration of BMSCs toward SDF-1 in an in vitro transwell system, and promoted the homing of BMSCs toward ischemic brain following intravenous administration in vivo. These beneficial effects of TMP were largely reversed by the CXCR4 antagonist AMD3100 in vitro and in vivo, further confirming that the SDF-1/CXCR4 axis regulates $\mathrm{BMSC}$ migratory behavior.

The mechanism of BMSC therapy for ischemic stroke primarily includes cell replacement, neuroprotection, angiogenesis, and neurogenesis [7, 34, 35]. The functional benefits after BMSC transplantation have been attributed to increased angiogenesis after ischemic stroke $[35,36]$. BMSCs promote angiogenesis through release of angiogenic factors and chemokines, such as vascular endothelial growth factor (VEGF), angiopoietin-1 (Ang1), basic fibroblast growth factor (bFGF), and SDF-1 [35-37]. SDF-1 not only facilitates the homing of transplanted BMSCs, but also increases vascular density and induces neovascularization in the ischemic tissue [37-39]. In the present study, we demonstrated that TMP preconditioning enhanced BMSC engraftation, upregulated SDF-1 expression, and promoted angiogenesis in the ischemic brain. In addition, the number of engrafted BMSCs was closely associated with angiogenesis after stroke. Our findings suggest that TMP preconditioning may enhance the mobilization of BMSCs into the ischemic brain, and engraftment of TMP-preconditioned
BMSCs promotes angiogenesis in the ischemic boundary zone. These changes significantly promoted the recovery of neurological function in rats with cerebral ischemia, which may have important clinical implications.

\section{Conclusions}

TMP preconditioning enhances the migration and homing ability of BMSCs and improves neurological performance after stroke. The combination of SDF-1 with CXCR4 may contribute to the trafficking of transplanted BMSCs. Our results suggest that TMP preconditioning enhances the neuroregenerative effects of BMSCs and could be a beneficial strategy to improve the therapeutic potential of cell transplantation for ischemic stroke in the clinical setting.

\section{Abbreviations}

BMSCs: Bone marrow-derived mesenchymal stem cells; BrdU: 5-Bromo-2deoxyuridine; BSA: Bovine serum albumin; CXCR4: CXC chemokine receptor 4; DMEM: Dulbecco's modified Eagle's medium; ECA: External carotid artery; FBS: Fetal bovine serum; GAPDH: Glyceraldehyde-3-phosphate

dehydrogenase; ICA: Internal carotid artery; MCAO: Middle cerebral artery occlusion; mNSS: Modified neurological severity score; MSCs: Mesenchymal stem cells; PBS: Phosphate-buffered saline; qRT-PCR: Quantitative reverse transcription-polymerase chain reaction; SD: Standard deviation; SDF-

1: Stromal cell-derived factor-1; TMP: Tetramethylpyrazine; vWF: Von Willebrand factor

\section{Acknowledgements}

The authors thank Dr Qinghua Sun for assisting in the preparation of this manuscript.

\section{Funding}

This study was supported by the National Natural Science Foundation of China (No. 81274113, 81073075) and the Public Welfare Technology Application Research Project of Zhejiang Province (No. 2016C33185).

\section{Availability of data and materials}

All data generated or analyzed during this study are included in this published article.

\section{Authors' contributions}

$L L, Y F, Y Y$ and $Y J Y$ were responsible for the performance of in-vivo experiments, data analysis, and manuscript preparation. TBQ, JPZ, and JJG were responsible for the performance of in-vitro experiments and data analysis. LSC contributed to the study design, data analysis and interpretation, and manuscript writing and revision. All authors read and approved the manuscript.

\section{Competing interests}

The authors declare that they have no competing interests.

\section{Consent for publication}

The authors declare that they consent to publication.

\section{Ethics approval}

All animal procedures were approved by the Experimental Animal Ethics Committee of Zhejiang Chinese Medical University (reference number: ZSLL-2014-37), Hangzhou, China and were conducted in accordance with the Guidelines for the Care and Use of Laboratory Animals established by Science and Technology Committee of Zhejiang Province.

\section{Publisher's Note}

Springer Nature remains neutral with regard to jurisdictional claims in published maps and institutional affiliations. 


\section{Author details}

'Department of Physiology, Zhejiang Chinese Medical University, Hangzhou 310053, China. ${ }^{2}$ Department of Anatomy, Zhejiang Chinese Medical University, Hangzhou 310053, China. ${ }^{3}$ Department of Pathology, Zhejiang Chinese Medical University, Hangzhou 310053, China.

Received: 15 November 2016 Revised: 23 March 2017

Accepted: 26 April 2017 Published online: 12 May 2017

\section{References}

1. Lees KR, Bluhmki E, von Kummer R, Brott TG, Toni D, Grotta JC, et al. Time to treatment with intravenous alteplase and outcome in stroke: an updated pooled analysis of ECASS, ATLANTIS, NINDS, and EPITHET trials. Lancet. 2010;375:1695-703.

2. Liu X, Ye R, Yan T, Yu SP, Wei L, Xu G, et al. Cell based therapies for ischemic stroke: from basic science to bedside. Prog Neurobiol. 2014;115:92-115.

3. Ikegame $Y$. Among mesenchymal stem cells: for the best therapy after ischemic stroke. Stem Cell Res Ther. 2013;4:9

4. Parekkadan B, Milwid JM. Mesenchymal stem cells as therapeutics. Annu Rev Biomed Eng. 2010;12:87-117.

5. Bang OY, Lee JS, Lee PH, Lee G. Autologous mesenchymal stem cell transplantation in stroke patients. Ann Neurol. 2005;57:874-82.

6. Lee JS, Hong JM, Moon GJ, Lee PH, Ahn YH, Bang OY. A long-term followup study of intravenous autologous mesenchymal stem cell transplantation in patients with ischemic stroke. Stem Cells. 2010;28:1099-106.

7. Chen J, Li Y, Wang L, Zhang Z, Lu D, Lu M, et al. Therapeutic benefit of intravenous administration of bone marrow stromal cells after cerebral ischemia in rats. Stroke. 2001;32:1005-11.

8. Chavakis E, Dimmeler S. Homing of progenitor cells to ischemic tissues. Antioxid Redox Signal. 2011;15:967-80.

9. Karp JM, Leng Teo GS. Mesenchymal stem cell homing: the devil is in the details. Cell Stem Cell. 2009;4:206-16.

10. Naderi-Meshkin H, Bahrami AR, Bidkhori HR, Mirahmadi M, Ahmadiankia N Strategies to improve homing of mesenchymal stem cells for greater efficacy in stem cell therapy. Cell Biol Int. 2015;39:23-34.

11. Marquez-Curtis LA, Janowska-Wieczorek A. Enhancing the migration ability of mesenchymal stromal cells by targeting the SDF-1/CXCR4 axis. Biomed Res Int. 2013;2013:561098.

12. Cencioni C, Capogrossi MC, Napolitano M. The SDF-1/CXCR4 axis in stem cell preconditioning. Cardiovasc Res. 2012;94:400-7.

13. Wang Y, Deng Y, Zhou GQ. SDF-1alpha/CXCR4-mediated migration of systemically transplanted bone marrow stromal cells towards ischemic brain lesion in a rat model. Brain Res. 2008;1195:104-12.

14. Shichinohe H, Kuroda S, Yano S, Hida K, Iwasaki Y. Role of SDF-1/CXCR4 system in survival and migration of bone marrow stromal cells after transplantation into mice cerebral infarct. Brain Res. 2007:1183:138-47.

15. Shen LH, Li Y, Chen J, Zacharek A, Gao Q, Kapke A, et al. Therapeutic benefit of bone marrow stromal cells administered 1 month after stroke. J Cereb Blood Flow Metab. 2007;27:6-13.

16. Hill WD, Hess DC, Martin-Studdard A, Carothers JJ, Zheng J, Hale D, et al. SDF-1 (CXCL12) is upregulated in the ischemic penumbra following stroke: association with bone marrow cell homing to injury. J Neuropathol Exp Neurol. 2004:63:84-96.

17. Kortesidis A, Zannettino A, Isenmann S, Shi S, Lapidot T, Gronthos S. Stromal-derived factor-1 promotes the growth, survival, and development of human bone marrow stromal stem cells. Blood. 2005;105:3793-801.

18. Son BR, Marquez-Curtis LA, Kucia M, Wysoczynski M, Turner AR, Ratajczak J, et al. Migration of bone marrow and cord blood mesenchymal stem cells in vitro is regulated by stromal-derived factor-1-CXCR4 and hepatocyte growth factor-c-met axes and involves matrix metalloproteinases. Stem Cells. 2006; 24:1254-64.

19. Wynn RF, Hart CA, Corradi-Perini C, O'Neill L, Evans CA, Wraith JE, et al. A small proportion of mesenchymal stem cells strongly expresses functionally active CXCR4 receptor capable of promoting migration to bone marrow. Blood. 2004;104:2643-5.

20. Liao SL, Kao TK, Chen WY, Lin YS, Chen SY, Raung SL, et al. Tetramethylpyrazine reduces ischemic brain injury in rats. Neurosci Lett. 2004;372:40-5.

21. Lin JB, Zheng CJ, Zhang X, Chen J, Liao WJ, Wan Q. Effects of tetramethylpyrazine on functional recovery and neuronal dendritic plasticity after experimental stroke. Evid Based Complement Alternat Med. 2015;2015:394926
22. Guo M, Liu Y, Shi D. Cardiovascular actions and therapeutic potential of tetramethylpyrazine (active component isolated from rhizoma chuanxiong): roles and mechanisms. Biomed Res Int. 2016;2016:2430329.

23. Kong X, Zhong M, Su X, Qin Q, Su H, Wan H, et al. Tetramethylpyrazine promotes migration of neural precursor cells via activating the phosphatidylinositol 3-kinase pathway. Mol Neurobiol. 2016:53:6526-39.

24. Sun HY, Fang MH, Ren $\mathrm{TH}$. Effect of ligustrazine on bone marrow hematopoiesis in mice after bone marrow transplantation. Zhongguo Zhong Xi Yi Jie He Za Zhi. 2002;22:365-8.

25. Zhang M, Gao F, Teng F, Zhang C. Tetramethylpyrazine promotes the proliferation and migration of brain endothelial cells. Mol Med Rep. 2014;10:29-32.

26. Longa EZ, Weinstein PR, Carlson S, Cummins R. Reversible middle cerebral artery occlusion without craniectomy in rats. Stroke. 1999;20:84-91.

27. Tsai LK, Wang Z, Munasinghe J, Leng Y, Leeds P, Chuang DM. Mesenchymal stem cells primed with valproate and lithium robustly migrate to infarcted regions and facilitate recovery in a stroke model. Stroke. 2011:42:2932-9.

28. Zhang L, Schallert T, Zhang ZG, Jiang Q, Arniego P, Li Q, et al. A test for detecting long-term sensorimotor dysfunction in the mouse after focal cerebral ischemia. J Neurosci Methods. 2002;117:207-14.

29. Chavakis E, Urbich C, Dimmeler S. Homing and engraftment of progenitor cells: a prerequisite for cell therapy. J Mol Cell Cardiol. 2008:45:514-22.

30. Tsai LK, Leng Y, Wang Z, Leeds P, Chuang DM. The mood stabilizers valproic acid and lithium enhance mesenchymal stem cell migration via distinct mechanisms. Neuropsychopharmacology. 2010;35:2225-37.

31. Honczarenko M, Le Y, Swierkowski M, Ghiran I, Glodek AM, Silberstein LE. Human bone marrow stromal cells express a distinct set of biologically functional chemokine receptors. Stem Cells. 2006;24:1030-41.

32. Yu X, Chen D, Zhang Y, Wu X, Huang Z, Zhou H, et al. Overexpression of CXCR4 in mesenchymal stem cells promotes migration, neuroprotection and angiogenesis in a rat model of stroke. J Neurol Sci. 2012;316:141-9.

33. Xie J, Wang H, Song T, Wang Z, Li F, Ma J, et al. Tanshinone IIA and astragaloside IV promote the migration of mesenchymal stem cells by upregulation of CXCR4. Protoplasma. 2013;250:521-30.

34. Chen J, Li Y, Katakowski M, Chen X, Wang L, Lu D, et al. Intravenous bone marrow stromal cell therapy reduces apoptosis and promotes endogenous cell proliferation after stroke in female rat. J Neurosci Res. 2003;73:778-86

35. Chen J, Zhang ZG, Li Y, Wang L, Xu YX, Gautam SC, et al. Intravenous administration of human bone marrow stromal cells induces angiogenesis in the ischemic boundary zone after stroke in rats. Circ Res. 2003;92:692-9.

36. Zacharek A, Shehadah A, Chen J, Cui X, Roberts C, Lu M, et al. Comparison of bone marrow stromal cells derived from stroke and normal rats for stroke treatment Stroke. 2010:41:524-30.

37. Shyu WC, Lin SZ, Yen PS, Su CY, Chen DC, Wang HJ, et al. Stromal cellderived factor-1 alpha promotes neuroprotection, angiogenesis, and mobilization/homing of bone marrow-derived cells in stroke rats. J Pharmacol Exp Ther. 2008:324:834-49.

38. Zhou B, Han ZC, Poon MC, Pu W. Mesenchymal stem/stromal cells (MSC) transfected with stromal derived factor 1 (SDF-1) for therapeutic neovascularization: enhancement of cell recruitment and entrapment. Med Hypotheses. 2007:68:1268-71.

39. Zhuang Y, Chen X, Xu M, Zhang LY, Xiang F. Chemokine stromal cellderived factor $1 /$ CXCL12 increases homing of mesenchymal stem cells to injured myocardium and neovascularization following myocardial infarction. Chin Med J (Engl). 2009;122:183-7.

\section{Submit your next manuscript to BioMed Central and we will help you at every step:}

- We accept pre-submission inquiries

- Our selector tool helps you to find the most relevant journal

- We provide round the clock customer support

- Convenient online submission

- Thorough peer review

- Inclusion in PubMed and all major indexing services

- Maximum visibility for your research

Submit your manuscript at www.biomedcentral.com/submit 